\title{
Eye Tracking- An Extensive Evaluation of a Contemporary and Ingenious Technology
}

\author{
Sparsh Rawlani \\ High School Graduate \\ St. Mary's Convent School \\ Ujjain, (M.P)
}

\author{
Purvesh Biyani \\ High School Graduate \\ Delhi Public School \\ Indore, (M.P)
}

\author{
Samarth Juneja \\ High School Graduate \\ St. Mary's Convent School \\ Ujjain, (M.P)
}

\begin{abstract}
When Google and Andy Rubin introduced the world with the first ever Android phone and a new concept in 2008, somewhere down the line, a group of researchers had curiosity about a technology that can enhance the life of human beings to a massive extent, which was to operate and manipulate thousands of things using the commands from our eyes. As if now, we are using high level technologies that are extremely easy to use, but anyone can imagine that operating our technological stuff with the power of our eyes and retina can be impressive. The devices that we use now-days contain a capacitive touch screen that detects the presence of our skin touch in order to get a command, however the process with the eye tracking is bit different. To have an instance of the eye-tracking technology, let us imagine a mobile game such as 'Fruit Ninja': a game where we cut fruits and vegetables by swiping left or right on our screen, so if we can score in the same game, not with the use of our hands, but with the directions from our eyes, than indeed the technology could be extraordinary.
\end{abstract}

This research paper will focus on the working and invention of this technology, and the devices in which its implementation could be possible. After the conclusion, the pros and cons and the futuristic scope of this technology would be stated in a detailed manner as per our research.

\section{Keywords}

The Eye Tribe, Formulation and development, reflection of light, infrared rays, calibration, tracking with pupil and cornea, pros \& cons, and future scope.

\section{INTRODUCTION}

The technology that we all operate now days are extremely advance, but introducing eye tracking in those advance technologies could result in a technology that would be doubly advance. Eye tracking in our day to day devises could have a devastating effect in our lives. It was around four years ago when a group of researchers decided to start a company, named "Eye Tribe" that could produce affordable eye tracking which can further be linked to our phones, tablets, and laptops for pursue an affordable eye tracking that can reduce our time and efforts by a great factor[1]. The eye tribe is a digital device which is quite long and can be attached to the bottom part of our traditional devices.

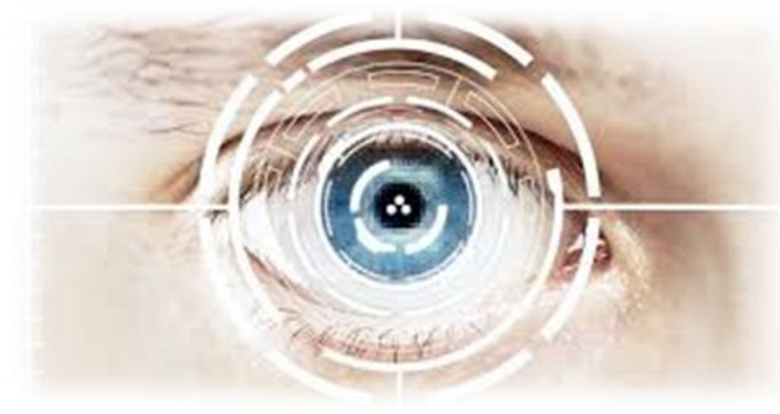

Fig 1: Eye Tracking Simulation

\subsection{Need for an Eye Tracking Device}

Eye Tracking was given a thought of implementation around seven years back and as a reason a group of Danish researcher began working on Eye Tribe. In these seven years, the world has witnessed a revolutionary change in the types of technical devices we all use today, but no one has ever encountered the level of simplicity that we can achieve with Eye-Tracking. Eye Tracking can reduce the complexity and can save our time by hours, but the method used to install devices like the Eye Tribe is quite tedious since not all the devices we use at our hands will support Eye-Tribe and the installation requires few lines of code, however, the code is quite simple and the device works effulgently after installation, therefore to reduce the complexities, Eye Tracking was implemented.[7]

\subsection{Eye Tribe Vs the Face-Unlock and Finger-Print Technologies:}

In the 18th year of the twenty-first century, the world has witnessed different types of technologies to secure our devices. Finger-print and Face-Unlock being the latest ones, where companies like Apple and One Plus have recently launched face unlock through true depth initialization of the device's front camera, companies like Samsung have already introduced Iris-Unlock feature, the sensor in the device registers the patters of the Iris and matches them each time you try to unlock your device. Although, the scenario would be quite dissimilar if Eye Tracking is implemented. To explain it in easier words, just imagine that to unlock your device, you just swipe the lock screen with the commands from your unique eyes, then indeed, Eye Tracking would be extremely fruitful and time saving. 


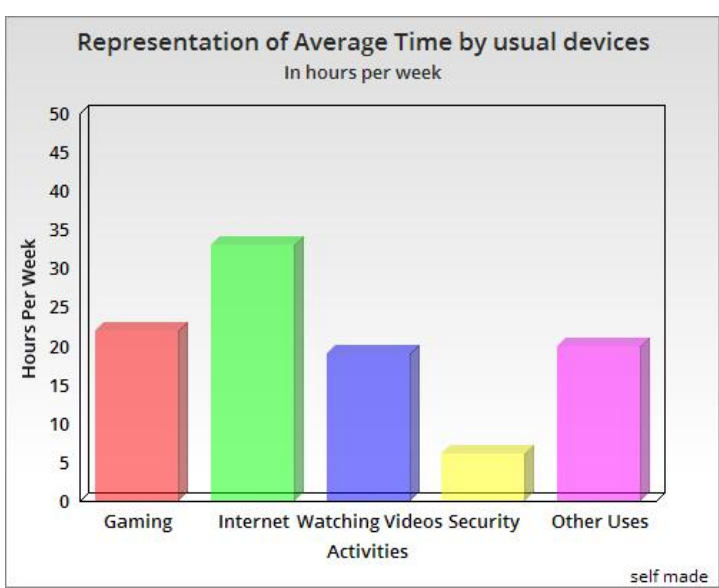

Fig.2: Average Time Consumed by usual Devises

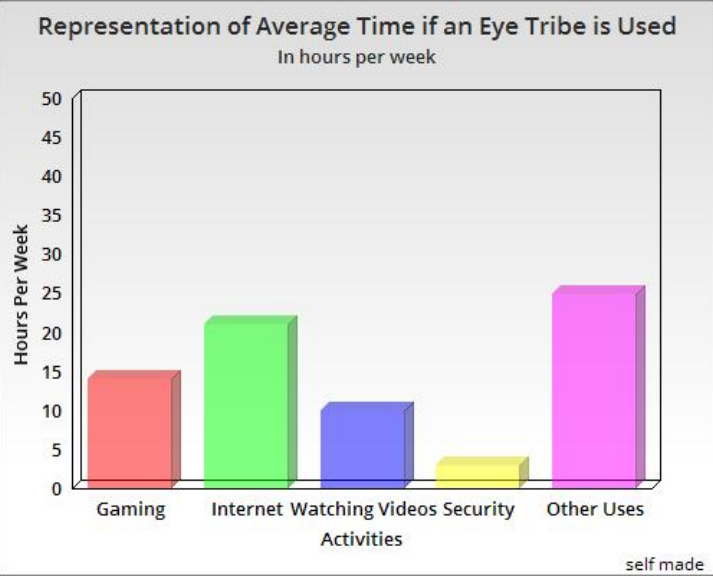

Fig.3: Average Time consumed with Eye Tribe

Eye tracking was implemented to navigate hands-free control on our personal devices including eye activated login, improved gaming experiences, cloud-based user engagement analytics and etc. The apparatus of an Eye Tracking Device such as Eye Tribe include a minute camera and an infrared LED to detect the signals from our eyes. Some introductory key-points about Eye Tribe and Eye Tracking are:

a) The Eye Tribe can be used with Mobiles and Tablets through a USB 3.0 cord, although the size still makes it difficult to use it with small devices.

b) The technology has not been implemented completely due to "Usability Testing", which means testing of the data captured by the eye and interpreting it, such interpretation is still unachievable by Eye Tracking.

c) Eye Tracking helps in providing an easy and valuable insight to the point where we focus our concentration.

d) Accuracy, peripheral vision, and the environmental setup affect the results of Eye Tracking by great amounts.

e) The next sophisticated steps will include the development of devices such as "Eye Tracking Glasses" that can replace the massive Eye Tribes.

\section{DEVELOPMENT AND FRAMEWORK OF EYE TRIBE}

In the year 2011, a group of 16 researchers started a project, which was to devise a device that could help people to achieve Eye Tracking in their electronic devices at affordable costs. The researchers induced a pack that included a tracker, a windows kit for developer and a USB charger. The Eye Tribe weighs $70 \mathrm{~g}$ on an average, has an operating range of 20-80 centimetres, and the dimensions are $20 \times 1.9 \times 1.9 \mathrm{~cm}$ approx. The devise works efficiently and has been undoubtedly used in scientific and educational purposes. [3]

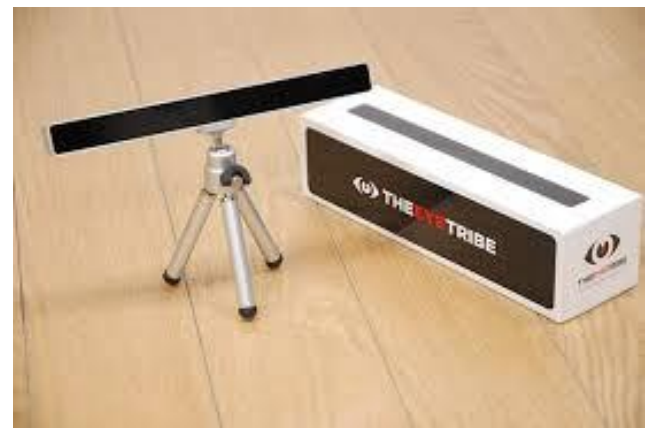

Fig 4: A look at the Eye Tribe

\subsection{How Does Eye Tribe/ Eye Tracking work?}

Few years back when Eye Tracking was implemented, it was also termed as "Gaze Interaction"(since Eye Tracking works either by locating the point of Gaze or by Tracking the motion of eyes, hence, locating the point of Gaze was termed as Gaze Interaction/Tracking). In easier words, the name suggests that Eye Tracking is used to locate the spot onto our screens with the help of devices such as Eye Tribe. In the first step, the "Illuminators" installed inside the Eye Tracker start emitting infrared rays that are reflected back by our eyes, in-fact, the rays are reflected by the pupil. In the next Step, a camera inserted into the Eye Tracker recognizes these reflections. After this, the reflected rays are captured and filtered by the camera and through the some algorithmic calculations, the Eye Tracker discover the position on the screen where the user is looking at and assigns the cursor or touch-point at that position precisely, which further results in an accurate Eye Tracking, although the process isn't that easy as it looks.[2]

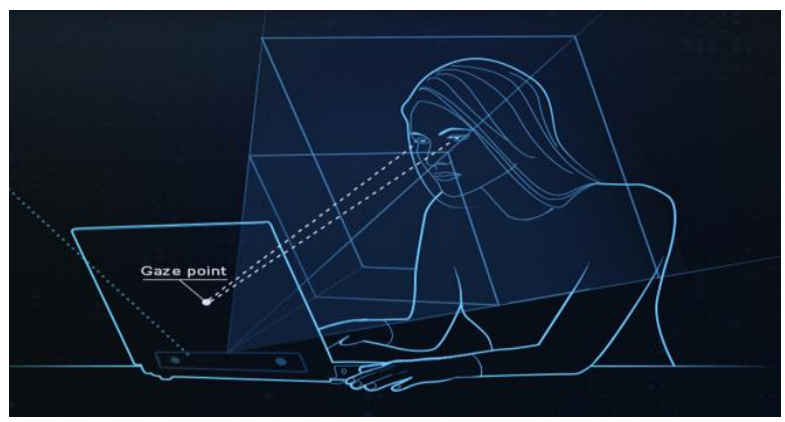

Fig 5: Tracking the Gaze Point

\subsubsection{Reflection of Infrared Rays through Eyes: Eye Tracking}

Since now, It is quite clear that to ensure precise and accurate Eye Tracking, the Eye Tracker should have advanced components that can result in appropriate tracking, and for that, the first step that is needed for Eye Tracking is the ideal reflection of infrared rays from the user's eyes. If the emitted 
infrared light from the infrared LED(Light Emitting Diode) inserted into the Eye Tracker, is not reflected properly, then the Tracker would not track the movement of our eyes.[4]

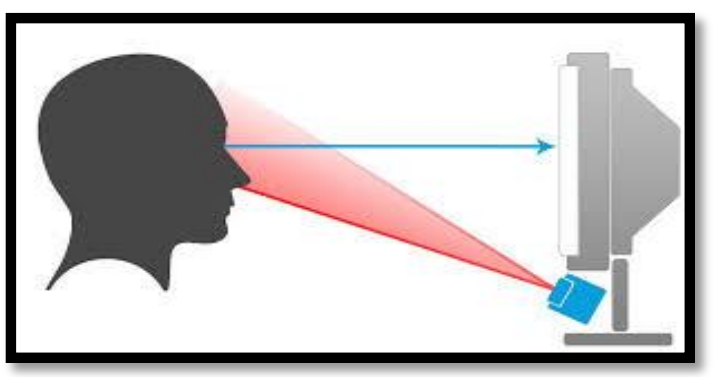

Fig 6: Capturing of Reflected Infrared rays by the Tracker

For this purpose, Eye Trackers have a unique element known as "Infrared Optometer". The Optometer was developed to calculate dynamic refractive power during the horizontal and vertical movements of our eyes.

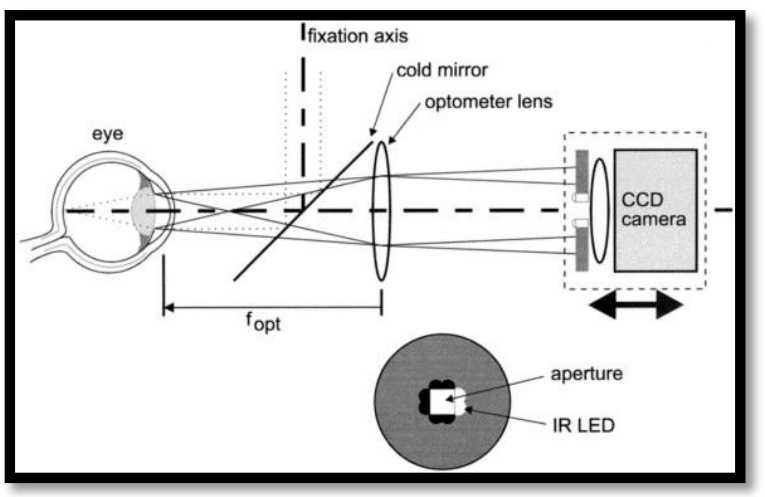

Fig 7: Design of an Infrared Optometer

The range of the Optometer is roughly -10 to $+10 \mathrm{D}$, refractive power is measured with an accuracy and precision of 0.41 and $0.05 \mathrm{D}$ respectively, and the maximum movement range within which the Optometer can locate the reflected light is approx 60 degrees.

\subsection{Calibration: Definition and Relevance with Eye Tracking}

As described earlier in this paper, that Eye Trackers work by two methods, either by locating the Gaze point or by Tracking our eye's motion, and therefore Calibration occurs when the Eye Tracker locates the "Point of Gaze" referred to as Gaze Interaction[5]. In other words, Calibration is the process in which the movements of the user's eyes are contemplated as the object from where a wholly customized gaze calculation takes place. This calibration procedure comprises of the measurement of the features of the eyes and, which further leads to the computation of the gaze data with the help of an Eye Model. In this process, we should look at the computer screen in order to help the Eye Tracker locate the points of Gaze easily. After the process concludes, the quality of the Calibration is represented by some green bars present on the front side of the Gaze Interactive Eye Tracker. Incomplete or inaccurate calibration leads to undesirable tracking, so to ensure the quality of Calibration and explain it to the people, the researchers introduced the world with types of Calibration.[10]

\subsubsection{Importance of Accuracy and Precision with Eye-Tracking}

After the implementation of Eye tracking into the real world, researchers encountered that Eye Tracking works differently with different people since all Eyes are distinctive and therefore accuracy and precision may differ with everyone's eyes. So to resolve this issue, different types of accuracy and precision were discovered and implemented into the Eye Trackers.[6]

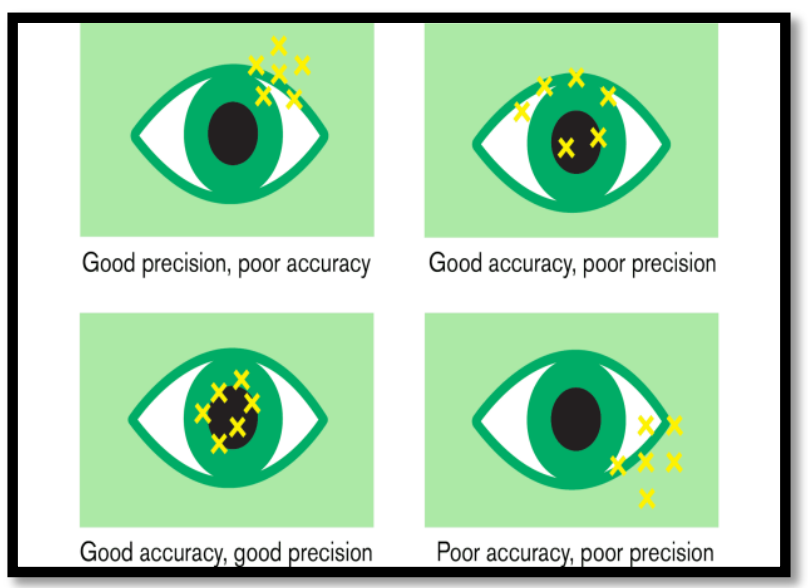

Fig 7: Types of Accuracy and Precision as presented by Tobii-Dynavox [9]

In terms of Eye Tracking, Accuracy and precision are defined in a manner that is quite different from the usual meaning of these words. Even Though, to some extent accuracy is related to accurateness of the measured gaze(point) position. It can be stated as the variation between the actual and the measured gaze position by the Eye Tracker, and Precision can be sated as the capability of the Eye Tracker to generate the same gaze measurement as estimated by the Eye Tracker and the difference incompletion of these lead to the types of Accuracy and Precision shown in Fig.7. Since accuracy and precision all are calculated in terms of gaze measurement, they are actually measured in terms of gaze angles initially(the angle difference between the two lines in the figure below represent the difference in accuracy), and the type of behaviour can be estimated from fig 7 afterwards. [13]

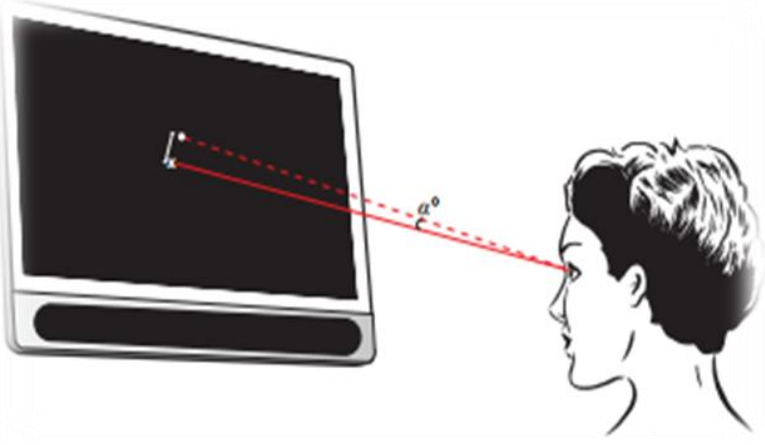

Fig 8: Estimation of Gaze angle for accuracy and precision

\section{EYE TRACKING WITH CLICKS}

For an Eye Tracker to work more accurately, it is quite important that the Eye Tracker should have the absolute information about our eyes. This is why calibration is necessary . At the time of calibration, the eye tracker notices how our eyes reflect light. The calibration is done with the 
help of a point, video or other graphic element that moves towards the screen. This calibrated figure is then coordinated with our exclusive 3D model of a human eye, and organised to give an supreme eye tracking experience.[11]

Nevertheless, there are simpler games and applications where precision is not as accurate, hence, calibration is required to execute Eye Tracking.

\subsection{Different Methods of Clicking (Initiating Eye Tracking)}

So as per the above explanations, it is quite clear that it is not possible to execute Eye Tracking without calibration in all the requisite cases, and therefore Calibration with the help of gaze interaction was introduced, and for calibration, it is important to know the methods of clicking that are used to obtain calibration. There are three methods specifically which are used to acquire gaze interaction through clicking, namely:-

\subsubsection{Blinking}

The process of Eye Tracking with the blink of an Eye is termed as Blinking, and it is possible to use blinking as a click to trigger the process of tracking, but it is not the finest way since it loses focus on the point at which you are focusing. [8]

\subsubsection{Dwelling}

Dwelling is the process of Eye Tracking which is opposite to Blinking. The click is performed without the blink of the Eye , and our eyes are focused on a specific area for a fixed number of milliseconds to ensure the click which can further initiate the process of Eye Tracking.[8]

\subsubsection{Switching}

If we are capable to control the switch with the Eye Tracker, it is actually the fastest and most proficient way of gaze interaction. We use gaze to choose the position for clicking, and then we push the switch on the Eye Tracker, to perform the click.[8]

\subsection{Pupil and Cornea tracking by the Eye Tracker}

Eye tracking has been typically used to interpret the visual attention of our eyes. There are abundant diverse procedures to recognize and track the motions of the eyes. Although, when it comes to remote, or non-invasive eye tracking, the most commonly used technique is the Pupil Centre Corneal Reflection (PCCR) [14]. It is defined as the usage of a light source to enlighten the eye causing highly visible reflections, and then a camera to apprehend an image of the eye viewing these replications. The camera imprisons the IR reflections and conducts Eye Tracking as explained in 2.1.

While using Pupil Centre Corneal Reflection Eye Tracking, two separate illuminating procedures are used, which are:

\subsubsection{Bright-Pupil Tracking}

All us have experienced "Red-Eye Error" issues while clicking a picture by a camera, using the flash light, a similar issue occurs while 'Bright Pupil Tracking'. During this, the illuminator is positioned nearer to the optical axis of the tracking device resulting the pupil to glow, causing a phenomenon that gives rise to Red Eyes in photos, and the solution to this issue is Dark-Pupil Tracking.[15]

\subsubsection{Dark-Pupil Tracking}

The case with 'Dark-Pupil Tracking' is exactly opposite to that of Bright Pupil Tracking. In this case, the illuminator is positioned far-away from the optical axis of the tracking device resulting in the appearance of the pupil to look gloomier as compared to the iris.

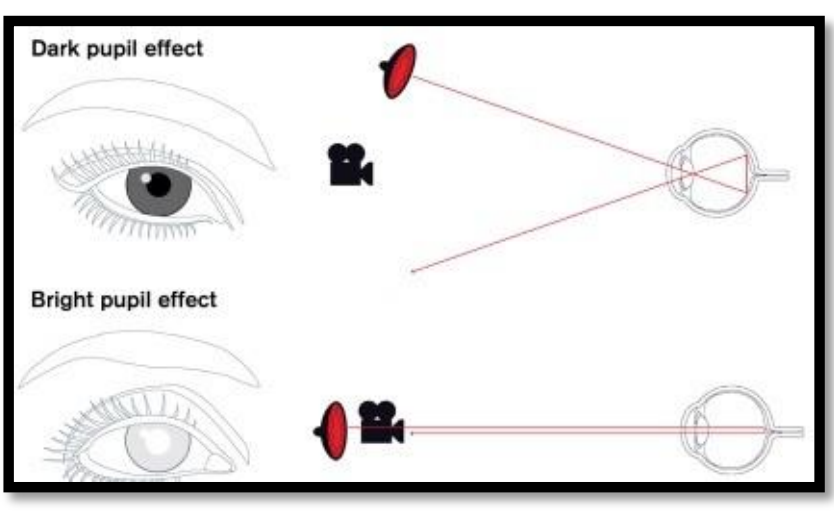

Fig 9: Bright and Dark-Pupil Tracking.

Certain people track better with dark pupil tracking rather than bright pupil tracking. Features like pupil size, age ,and environmental lights affect the methods of Eye Tracing to a great extent. Eye trackers are unique in the way they smoothly toggle between bright and dark pupil tracking, so you will always have an improved eye tracking involvement. It is essentially the association between the pupils and the glints(small parts of reflected light), or reflections, that help the eye tracker regulate where you are looking.[15]

\section{TYPES OF EYE TRACKING}

The sections 2.1 and 2.2 of this paper explain us about the two best ways in which Eye Tracking works, namely Reflection of IR rays, and Calibration. The type of Eye Tracking differs in both of these conditions.

There are two types of widely used Eye Tracking methods:[12]
A. Screen Based Eye Tracking
B. Eye Tracking through ET(Eye Tracking) Glasses

\subsection{Screen based Eye Tracking}

Screen-based devices require the users to interact with the entire monitor and screen-based content, and therefore it is the best and the most practical setup for observations of two and three model structures. The Eye Tracker attached to the bottom part of our screens capture the reflection of IR rays from our eyes and initiates precise tracking. This method is also helpful in cases where the user needs to move the device freely. It is the best of the two methods because of compatibility, although results may vary as per the quality of the system.

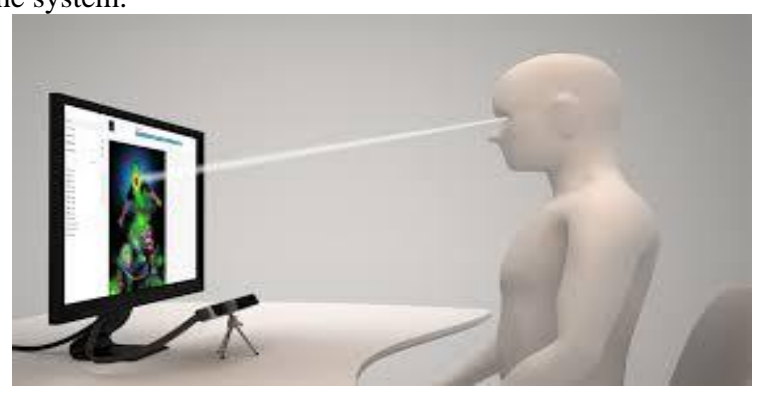

Fig 10: Screen Based Eye Tracking 


\subsection{Eye Tracking through ET Glasses:}

The second type of Eye Tracking devices, which result in remote or wireless Eye Tracking are Eye Tracking glasses. Since many people around the world use optical lenses and glasses, implementing eye tracking within the glasses by designing them in a unique manner was indeed an excellent thought. Even the Eye Tracking glasses use the PCCR (pupil centre corneal reflection) technique to track the motion and directions given by our eyes. The fixed-in camera to the frame of the glasses captures the reflections of the infrared light which is emitted from another built-in device within the specs. After the recognition of the light that was reflected from the pupil, some improvised sensors calculate the gaze direction and establish accurate Eye Tracking with calibration. Since Calibration is not the finest way to commence Eye Tracking, the ET Glasses may result in incomplete tracking.[17]

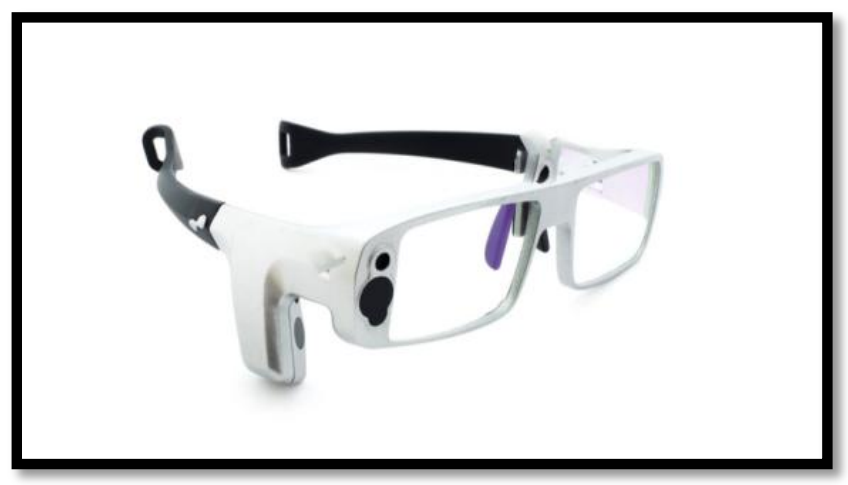

Fig 11: Eye Tracking Glasses

\section{ADVANTAGES}

The abstract of this paper clearly outcastes the fact that Eye Tracking leads to richer and more independent life, and therefore it has multiple advantages:

\section{A. Speech Generation and Communication \\ Eye Tracking is indeed an important technology for those having single or multiple disabilities. People who are unable to speak, use their fingers, hands or any other body part in a genuine manner to operate a keyboard, mouse or touch-screen can use eye tracking to operate such devices with their eyes. Eye Tracking makes it easier to connect with the world for such people.}

\section{B. Contemporary Design}

Unlike other track boxes, eye tracker has a contemporary design. The eye tribe does not require a separate power source, this allows the user to comfortably carry the eye tribe eye tracker anywhere along. The device uses a USB 3.0 connection, which allows it to run with most computers and tablets.

It usually weighs around 90-100 grams and is therefore easy to assemble.

\section{One time calibration}

The eye tracking device requires only one time calibration. While calibrating, it remembers how the user responds to the system and saves it for later. There is no hardware to deal with it, we simply need to install the PCI card in our computer and load the software instantly.

\section{Environmental control}

Numerous appliances, such as TVs, AC's and even doors, can be manipulated by IR remote controls. Users with restricted movements, such as those with an injury, can use their eye- tracking as a remote control and function appliances via gaze interaction.

\section{E. Unique 3d Eye-Model}

Earlier in this paper, it was mentioned that an Eye-model is used in the Eye Tracker to calculate the gaze data, in-order to begin calibration. In these Eye-Models, a unique update was introduced with which we can initiate calibration for EyeTracking even when our head or neck is not in a still position. However, it may result in incomplete tracking in some cases, but it is far the best model to interpret gaze data.

\section{DISADVANTAGES}

Anything in this universe, whether living or non-living, food or technology, has an unwanted disadvantage, since nothing is perfect in this world. So is the fact with the unconventional technologies like Eye Tracking, even though it can be a boon for people with disorders, it can actually be a drawback due to certain issues, and some of them are described below:

\section{A. Imperfect Tracking with Eye Glasses/ Contact Lenses} Anyone suffering from disorders like Myopia or Hyperopia would have to use vision correction aid like eyeglasses or contact lenses. The reflections created from such aids will interfere with the tracker's ability to track accurately and thus interfere with the system. Hence, it is extremely difficult for such individuals to use Eye Trackers.

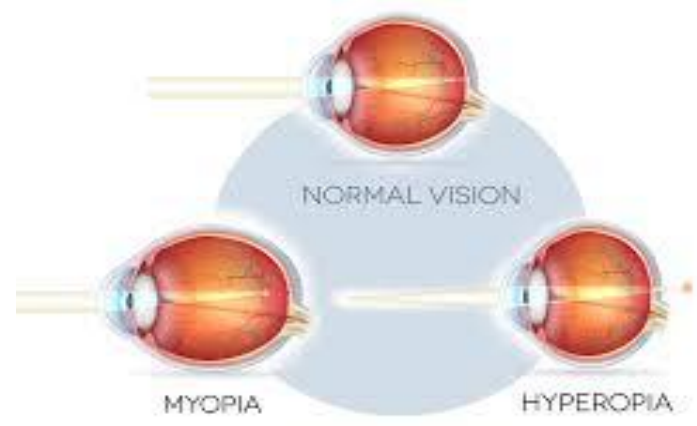

Fig 12: Normal Vision Compared to Myopia and Hyperopia, which can affect Eye Tracking

B. Faulty Tracking with Sunlight and False Reflections Apart from people with vision disorders, even people with no disorder can have a tough time using the eye tracker. There can be false reflections in the cornea of the user's eye, if there is a second lighting or sunlight in the room, then this can lead to interpretational faults. The Eye Tracker reflects IR rays from our Eyes and in the presence of lights with strong wavelengths such as sunlight, the camera that is supposed to capture the IR rays, may capture the sunlight and hence can result in false reflections and faulty tracking.

\section{Unsuccessful Calibration due to Unsteady Movement of} Eyes

In the complete process of Eye Tracking, the user's Eye acts as an input(similar to what a mouse acts for a computer), and moving our eyes is an entire unintentional act, therefore it is quite difficult to roll our eyes precisely as per the requirement. Instability can occur if the tracker fails to obtain an steady image of our Eye due to continuous blinking or unstable head, which can further result in misinterpretation and unsuccessful calibration.

\section{Expensive Equipments}

One of the major cons Eye Tracking is its expensive setup and its equipments. Although some companies are selling Eye 
Tracker for an affordable price, but the entire set-up may costs thousands of dollars. Even a company like Google with a total brand value of 245 billion\$, has only one lab for the research and execution of Eye Tracking. Secondly, even if you are able to afford all the equipments, you will have to spend money training people on how to use that, which doesn't sound an inexpensive option. Even if the price of the Eye Tracker may sound affordable after looking at its benefits, the entire equipmental setup might cost up to 5000-10000 US dollars.

\section{CONCLUSION}

It was the year 2011, when four researchers thought of implementing the long awaited technology, "Eye Tracking", and established a company named 'Eye Tribe' and began to manufacture useable Eye Trackers for common people. At present, the Eye Tribe has 16 members and has made an Eye Tracking Tablet which is available at a cost less than other Eye-Trackers. The sole purpose of developing a technology like Eye Tracking was to help individuals who have a disability or disorder and due to which they are unable to communicate with usual methods. After researching on how is Eye Tracking possible, we found two executable methods which are briefly explained in this paper. The first one was to reflect the IR rays through the user's eye and then capture those rays with the help of a camera inserted in the Eye Tracker, and the other was to calculate and interpret the gaze data with the help of a $3 \mathrm{~d}$ eye model that can execute calibration. Both of the methods proved to be fruitful and both of them have multiple pros and cons as well. After that, we saw three ways to initiate Eye Tracking(ways of clicking), Blinking, Dwelling, and Switching, Switching being the fastest possible way to ensure tracking. However, in cases of remote Eye Tracking, a technique known as PCCR was implemented which was further distinguished into Bright and Dark-Pupil tracking. Even though there were several methods and devices for conducting Eye Tracking, it has numerous disadvantages, cost being the initial one, and vision aid being the scientific one. To operate a device with our eyes accurately and precisely sounds pretty amazing and easy, but is actually extremely tricky and tedious, since it is not possible to keep track of our eyes all the time. At last, we have mentioned some future enhancements as per our research, which could make Eye Tracking unproblematic and affordable in future.

\section{FUTURE SCOPE AND POSSIBLE ENHANCEMENTS}

Since we have figured out the most reasonable and scientific disadvantages of Eye Tracking, it is necessary to outcast some possible solutions and futuristic enhancements of Eye Tracking that can make it possible for every individual to use and afford this foremost technology, and therefore here are some possible upgrades for Eye Tracking[16]:

\subsection{Operating Physical Devices with Eye Tracking}

The present Eye Trackers are only limited to electronic devices like mobiles and computers and are entirely missing in physical or automatic devices. One of the biggest purpose of Implementing Eye Tracking was to help disabled people who are unable to use their body parts fully. To solve this issue, one of the biggest enhancement would be to implement Eye Tracking in vehicles and medical devices such as wheelchairs. For a person who cannot walk, eye tracking being introduced in his wheelchair could be extremely helpful, and this can be achieved if the Eye Trackers slotted in the wheelchairs and vehicles could track our gaze point in an efficient and smart way.

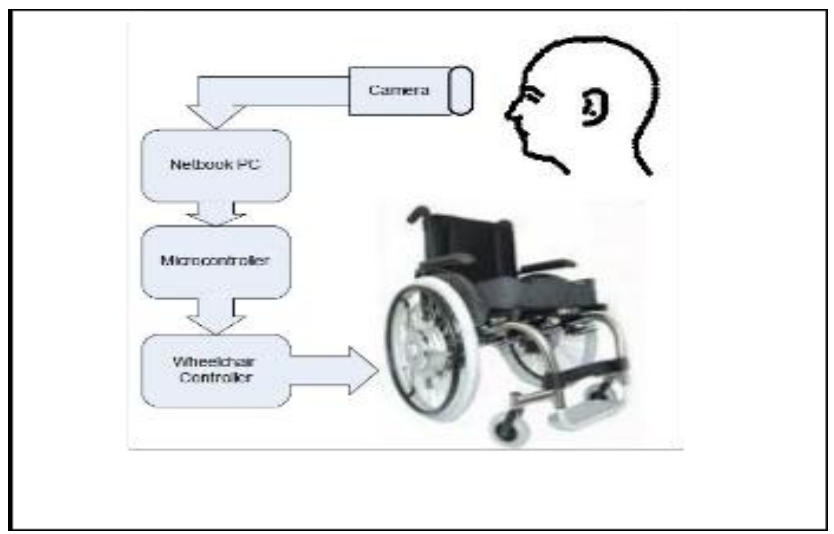

Fig 13: Eye Tracking in Wheelchairs

\subsection{Open source Software for ET setup}

At present, all the Eye Tracking systems around the world work only on proprietary software(a software that is not free and its source code and rights are with the manufacturer), which means that for every controllable action with Eye Tracking, we will need to write a separate application for executing the required task. In order to get rid of this, it is important to have an Open Source Software through which applications can be executed by anyone, since only the manufacturer can develop it if the software used is proprietary. Once an open source software is implemented, eye tracking would become easier in-terms of execution.

\subsection{Integration}

Another possible development would be the integration of Eye Tracking with "Motion Capture Data", which will assist us to examine the synchronization between our Eyes and different body parts. In simpler words, it would help us to visualize the real time data or activities, which will further give us a visual approach an individual's body movements. This may sound astonishing and impossible, but this is what researchers are trying to achieve with Eye Tracking by integrating it with motion capture data.

\subsection{Affordable and Accommodative Set-Up}

A recent article on Eye Tracking highlighted that the entire setup at a laboratory in London, cost about $20000 £$, and for a person with disorders, it is not an affordable and practical option. Since it is important to detect even a tiny moment of our eye, the setup becomes so large that it requires a separate laboratory for eye tracking to be executed precisely. The high resolution is another reason that forces the developers to increase the size of Eye Trackers, and to get rid of size and cost , an Eye Tracker needs to be developed that can work on low resolution at a cheaper rate.

\section{REFERENCES}

[1] Hayoe, M., \& Ballard, D. (2005). Eye movements in natural behaviour. Trends in Cognitive Science, 9, 188 194.

[2] Mansour, Jamal K., and Heather D. Flowe. "What is eye tracking?- Eye Tracking and Eye Witness memories, academia.edu.

[3] Duchowski, Andrew T. "Eye tracking methodology." Theory and practice 328 (2007). 
[4] Jacob, Robert JK, and Keith S. Karn. "Eye tracking in human-computer interaction and usability research: Ready to deliver the promises." The mind's eye. 2003. 573-605.

[5] Holmqvist, Kenneth, Marcus Nyström, Richard Andersson, Richard Dewhurst, Halszka Jarodzka, and Joost Van de Weijer. Eye tracking: A comprehensive guide to methods and measures. OUP Oxford, 2011.

[6] Ooms, Kristien, Lien Dupont, Lieselot Lapon, and Stanislav Popelka. "Accuracy and precision of fixation locations recorded with the low-cost Eye Tribe tracker in different experimental setups." Journal of eye movement research 8 , no. 1 (2015).

[7] Krafka, K., Khosla, A., Kellnhofer, P., Kannan, H., Bhandarkar, S., Matusik, W., \& Torralba, A. (2016). Eye tracking for everyone. arXiv preprint arXiv:1606.05814.

[8] Kyungdae, P. A. R. K., K. A. N. G. Jiyoung, K. O. H. Sanghyuk, P. A. R. K. Mijung, O. H. Saegee, and L. E. E. Chihoon. "Method for operating user functions based on eye tracking and mobile device adapted thereto." U.S. Patent 9,092,051, issued July 28, 2015.

[9] Gibaldi, A., Vanegas, M., Bex, P. J., \& Maiello, G. (2017). Evaluation of the Tobii EyeX Eye tracking controller and Matlab toolkit for research. Behavior research methods, 49(3), 923-946.

[10] Hutchinson, Thomas E. "Eye movement detector with improved calibration and speed." U.S. Patent No. 4,950,069. 21 Aug. 1990.
[11] Morimoto, Carlos H., and Marcio RM Mimica. "Eye gaze tracking techniques for interactive applications." Computer vision and image understanding 98, no. 1 (2005): 4-24.

[12] Holmqvist, Kenneth, Marcus Nyström, Richard Andersson, Richard Dewhurst, Halszka Jarodzka, and Joost Van de Weijer. Eye tracking: A comprehensive guide to methods and measures. OUP Oxford, 2011.

[13] Morimoto, Carlos H., and Marcio RM Mimica. "Eye gaze tracking techniques for interactive applications." Computer vision and image understanding 98.1 (2005): 4-24.

[14] Kassner, Moritz, William Patera, and Andreas Bulling. "Pupil: an open source platform for pervasive eye tracking and mobile gaze-based interaction." Proceedings of the 2014 ACM international joint conference on pervasive and ubiquitous computing: Adjunct publication. ACM, 2014.

[15] Morimoto, C. H., Koons, D., Amir, A., \& Flickner, M. (2000). Pupil detection and tracking using multiple light sources. Image and vision computing, 18(4), 331-335.

[16] Schiessl, M., Duda, S., Thölke, A., \& Fischer, R. (2003). Eye tracking and its application in usability and media research. MMI-interaktiv Journal, 6, 1-10.

[17] Babcock, Jason S., and Jeff B. Pelz. "Building a lightweight eyetracking headgear." Proceedings of the 2004 symposium on Eye tracking research \& applications. ACM, 2004. 